\title{
RISCO DE LESÃO POR PRESSÃO EM IDOSOS COM COMPROMETIMENTO NA REALIZAÇÃO DE ATIVIDADES
} DIÁRIAS

\section{RISK OF PRESSURE INJURY IN ELDERLY INDIVIDUALS WITH COMPROMISSE IN DAILY ACTIVITIES}

\section{RIESGO DE LESIÓN POR PRESIÓN EN ANCIANOS CON COMPROMISO EN LA REALIZACIÓN DE ACTIVIDADES COTIDIANAS}

Vanete Aparecida de Souza Vieira ${ }^{1}$, Mariana Diniz Costa Santos ${ }^{1}$, Amanda do Nascimento Almeida ${ }^{1}$, Cristiane Chaves de Souza $^{2}$, Mariana Ferreira Vaz Gontijo Bernardes ${ }^{3}$, Luciana Regina Ferreira da Mata ${ }^{4}$

\section{RESUMO}

Objetivo: avaliar o risco de idosos institucionalizados com comprometimento na realização das atividades de vida diárias (AVDs) desenvolverem lesão por pressão (LP). Método: trata-se de estudo transversal quantitativo, com amostra de 44 idosos, que utilizou como instrumentos de coleta de dados: questionário sobre perfil sociodemográfico dos idosos, escala de Katz e escala de Braden. Os dados foram analisados no programa estatístico SPSS 17.0. Resultados: verificou-se que 64,3\% dos idosos possuíam risco de desenvolver LP, e 57,1\% eram dependentes para realizar cinco ou mais AVDs. Quanto maior a independência para desenvolvimento de AVDs, menor o risco de desenvolver UP $\left(r_{s}=-0,74 ; p<0,05\right)$. Conclusão: a utilização de escalas preditivas como as de Braden e de Katz proporciona parâmetros para o enfermeiro planejar cuidados com a pele de modo individualizado, visando a segurança e bem-estar dos idosos institucionalizados.

Descritores: Segurança do paciente; Lesão por pressão; Idoso; Institucionalização; Enfermagem.

\section{ABSTRACT}

Objective: to evaluate the risk of institutionalized elderly people with impairment in performing daily life activities (DLAs) to develop pressure injury (PI). Method: This is a cross-sectional quantitative study, with a sample of 44 elderly people, which used as data collection instruments: a questionnaire on the sociodemographic profile of the elderly people, Katz scale and Braden scale. The data were analyzed in the statistical program SPSS 17.0. Results: it was verified that $64.3 \%$ of the elderly people had a risk of developing $\mathrm{PI}$, and $57.1 \%$ were dependent to perform five or more DLAs. The higher the independence for DLA development, the lower the risk of developing PU $\left(r_{s}=-0.74, p<0.05\right)$. Conclusion: The use of predictive scales such as those of Braden and Katz ones provides parameters for the nurse to plan individualized skin care, aiming at the safety and well-being of the institutionalized elderly people.

Descriptors: Patient safety; Pressure ulcer; Aged; Institutionalization; Nursing.

\section{RESUMEN}

Objetivo: evaluar el riesgo de ancianos institucionalizados con comprometimiento en la realización de las actividades de vida diaria (AVDs) desarrollar lesión por presión (LP). Método: se trata de un estudio transversal cuantitativo con una muestra de 44 ancianos que utilizó como instrumentos de recolección de datos: cuestionario sobre perfil sociodemográfico de los ancianos, escala de Katz y escala de Braden. Se analizaron los datos en el programa estadístico SPSS 17.0. Resultados: se verificó que el $64,3 \%$ de los ancianos tenían riesgo de desarrollar LP, y el 57,1\% era dependiente para realizar cinco o más AVDs. Cuanto mayor la independencia para el desarrollo de AVD, menor es el riesgo de desarrollar UP ( $r s=-0,74 ; p<0,05)$. Conclusión: la utilización de escalas predictivas como las de Braden y de Katz proporciona parámetros para el enfermero planificar atención con la piel de modo individualizado, buscando la seguridad y el bienestar de los ancianos institucionalizados.

Descriptores: Seguridad del paciente; Úlcera por presión; Anciano; Institucionalización; Enfermería.

${ }^{1}$ Graduada em Enfermagem pela Universidade Federal de São João del Rei. ${ }^{2}$ Graduada em Enfermagem. Doutora em Enfermagem pela Universidade Federal de Minas Gerais. Docente na Universidade Federal de Viçosa. ${ }^{3}$ Graduada em Enfermagem. Mestre em Enfermagem pela Universidade Federal de São João Del Rei. ${ }^{4}$ Graduada em Enfermagem. Doutora em Enfermagem pela Escola de Enfermagem de Ribeirão Preto da Universidade de São Paulo. Docente na Universidade Federal de Minas Gerais.

Como citar este artigo:

Vieira VAS, Santos MDC, Almeida NA, et al. Risco de lesão por pressão em idosos com comprometimento na realização de atividades diárias. Revista de Enfermagem do Centro-Oeste Mineiro. 2018;8:e2599. [Access ]; Available in: DOI: http://dx.doi.org/10.19175/recom.v7i0.2599 


\section{INTRODUÇÃO}

A lesão por pressão (LP) é uma lesão de pele e/ou tecidos moles resultante de hipóxia celular, que pode provocar necrose tecidual como resultado de pressão ou pressão com fricção e cisalhamento, podendo apresentar-se em pele íntegra ou como úlcera aberta. É encontrada sobre proeminência óssea ou relacionada ao uso de dispositivo médico ou a outro artefato. Sua ocorrência está relacionada a uma diminuição da qualidade de vida do sujeito, uma vez que pode estar associada a sintomas como dor e deformidade $^{(1)}$.

A cada ano, nos Estados Unidos, identificase mais de 2,5 milhões de pessoas com LP nas mais diversas configurações de saúde, sendo $0,4 \%$ a $38 \%$ nos cuidados agudos, $0 \%$ a $17 \%$ nos cuidados domiciliares e $2 \%$ a $24 \%$ em instituições de longa permanencia para idosos (ILPI) $)^{(1-2)}$. Cerca de 60.000 pacientes morrem como resultado direto de uma LP a cada ano ${ }^{(2)}$. Desta forma, a prevalência de LP tornou-se uma grande ameaça para a saúde pública e para o sistema de saúde dos EUA ${ }^{(2)}$. Já no Reino Unido os casos novos de LP ocorrem entre $4 \%$ a $10 \%$ dos pacientes admitidos em instituição hospitalar ${ }^{(3)}$. No Brasil, embora existam poucos trabalhos robustos sobre incidência e prevalência de LP, as ocorrências dessas são altas, variando de $10,6 \%$ a $62 \%$, enquanto a variação desta é de $7,3 \%^{(4)}$. Esta grande diferença é justificada pelo perfil da população estudada, metodologia adotada e por incluir ou não as LP de estágio $1^{(1)}$.

As LP estão relacionadas ao aumento da expectativa de vida global, sendo os pacientes geriátricos e com restrição de movimento os mais afetados por essa condição ${ }^{(3)}$. 0 envelhecimento populacional e a institucionalização de idosos são fenômenos crescentes na população brasileira. Quando não se envelhece de maneira saudável, o aumento da longevidade e do processo incapacitante aumenta a prevalência de LP, ocasionando mais um problema de saúde para o idoso $^{(5)}$. Denominamos processo incapacitante quando uma determinada condição (aguda ou crônica) afeta a funcionalidade dos idosos comprometendo suas atividades cotidianas, as quais, em termos de avaliação em saúde, são conhecidas como atividades de vida diária $(A V D s)^{(6)}$. Em idosos que vivem em ILPI, a LP é um problema de saúde, sobretudo para aqueles que passam maior parte do tempo acamados ou sentados e com exposição a fatores extrínsecos (fricção, cisalhamento e umidade) e intrínsecos (desnutrição, envelhecimento, baixa pressão arteriolar, perda da sensibilidade, diminuição da força muscular ou mobilidade, incontinência, hipertermia, anemia e tabagismo $)^{(7)}$.

Estudo realizado numa ILPI brasileira mostrou uma prevalência alta de LP nos idosos institucionalizados. Alguns desafios para implementar ações para reduzir a ocorrência da LP nessa população, no local estudado, foram identificados: quantitativo reduzido de profissionais da equipe de enfermagem para a assistência de idosos com severo grau de dependência, uso precário de materiais de higiene pessoal, nutrição inadequada, a dificuldade para a introdução de protocolos e escalas de classificação e tratamento das lesões, e a falta de treinamento e de motivação para o cuidado continuado ${ }^{(8)}$.

Dentre os profissionais que prestam assistência ao idoso e que podem atuar na prevenção da ocorrência da LP, destacam-se os profissionais de enfermagem, que podem realizar o tratamento e a prevenção de lesões, motivo pelo qual esta temática tem se tornado alvo de pesquisas na área ${ }^{(3)}$. Nesse contexto, o enfermeiro dispõe de escalas validadas capazes de predizer o risco de desenvolvimento de LP e colaborar na prevenção das mesmas por meio da identificação precoce dos indivíduos em risco ${ }^{(1,9)}$.

Embora a ação de avaliar o risco de idosos institucionalizados desenvolverem LP seja de suma importância para enfermagem e, ainda, a prevalência de LP tenha se apresentado alta nas ILPI, percebe-se uma carência de estudos nacionais publicados que abordem o risco de idosos institucionalizados desenvolverem LP em idosos com algum comprometimento para realizar as AVDs. Percebe-se ainda que, em ILPI, os enfermeiros nem sempre utilizam instrumentos padronizados para identificar pacientes em risco de desenvolver LP e propor medidas preventivas, o que pode comprometer a assistência prestada ${ }^{(8)}$. Entretanto, é imprescindível que o profissional da enfermagem atue com o desígnio de prevenir o surgimento das $L P$, visto que o aparecimento das mesmas pode diminuir a autonomia do idoso, além de o expor a risco de complicações advindas do comprometimento da integridade da pele. Para isso, o profissional pode utilizar recursos como instrumentos padronizados que possibilitem identificar pacientes em risco de desenvolver LP e, assim, otimizar medidas preventivas, 
contribuindo para a manutenção do estado de saúde dos idosos.

Considerando este contexto, formulou-se para o estudo a seguinte questão norteadora: Qual é o risco de idosos institucionalizados com comprometimento na realização das AVDs desenvolverem LP? Destarte, objetivou-se avaliar o risco de idosos institucionalizados com comprometimento na realização das atividades de vida diárias (AVDs) desenvolverem lesão por pressão (LP).

\section{MÉTODOS}

Trata-se de estudo transversal, realizado em três ILPI de um município do interior de Minas Gerais. A dinâmica de cuidados com os idosos nas instituições é semelhante, todas possuem cuidadores responsáveis pelos cuidados com os idosos em tempo integral. A população do estudo foi composta por 114 idosos, que corresponde ao total de idosos institucionalizados no período da coleta de dados. Foram considerados critérios de inclusão: ser residente na ILPI há, no mínimo, dois meses; ter idade superior a 60 anos; apresentar o diagnóstico de enfermagem "Mobilidade física prejudicada", avaliado conforme os fatores relacionados e características definidoras descritos na taxonomia da NANDA- ${ }^{(10)}$. Cabe ressaltar que foram incluídos apenas os idosos com "Mobilidade Física prejudicada", pois esses, necessariamente, apresentavam algum grau de comprometimento na realização das AVDs, o que era necessário para responder à pergunta de pesquisa. Foram excluídos da pesquisa os idosos que já possuíam LP quando da primeira avaliação dos pesquisadores e os que estavam em situação de abandono e não possuíam representante legal, perfazendo uma amostra final de 42 idosos.

A coleta de dados foi realizada por três pesquisadores no período de março a maio de 2014, utilizando os seguintes instrumentos: questionário perfil sociodemográfico do idoso, Escala de Katz e Escala de Braden ${ }^{(11-12)}$.

O questionário sociodemográfico elaborado pelos autores contemplou questões abertas e fechadas para levantamento de dados referentes à idade, sexo, cor da pele, religião, situação conjugal, número de filhos, custeio da internação, tempo de internação, escolaridade.

A escala de Katz avalia o grau de dependência/independência do idoso para realização das AVDs: capacidade de tomar banho, vestir-se, ir ao vaso sanitário, transferir-se, manter-se continente e alimentar-se. É uma escala graduada de zero a seis, sendo: zero - não apresenta dependência em nenhuma das atividades, seis - apresenta alteração nas seis atividades. Quanto maior a pontuação maior o nível de dependência ${ }^{(11)}$.

A escala de Braden é composta por seis itens que objetivam avaliar o risco para desenvolvimento de LP, conforme pontuaçã̃o: 19 a 23 pontos, sem risco; 15 a 18 pontos, baixo risco; de 13 a 14, risco moderado; de 10 a 12, alto risco; e 9 ou menos pontos, risco muito elevado ${ }^{(12)}$.

Previamente ao início da coleta de dados, os pesquisadores receberam capacitação acerca da utilização da escala de Braden a partir da avaliação de casos clínicos fictícios previamente validados por especialistas quanto ao conteúdo. No total, 12 casos foram elaborados pela pesquisadora responsável e enviados para avaliação de três peritos com experiência em ensino, pesquisa, e utilização da escala de Braden na prática clínica. Foram selecionados para o treinamento, sete casos, que apresentaram concordância em $100 \%$ dos seguintes itens avaliados entre os três peritos: nível de risco na escala de Braden; caso que permita a aplicação da escala de Braden; redação do caso clínico clara, de modo a permitir avaliação acurada das subescalas da escala de Braden; pistas clínicas claras, de modo a identificar a pontuação em cada item da escala; adequação dos estudos de caso para serem aplicados a alunos do sétimo período do curso de enfermagem para fins de treinamento na utilização da escala de Braden. Para aplicação da escala de Braden, foram selecionadas duas pesquisadoras que alcançaram concordância excelente com os peritos (Kappa $\geq$ $0,90)^{(13)}$.

Cabe ressaltar que a aplicação das escalas de Katz e de Braden foram realizadas por pesquisadores independentes, de modo a garantir a blindagem dos avaliadores.

Para análise descritiva dos dados, utilizaram-se tabelas de distribuição de frequência. Foi realizado o cálculo da prevalência de idosos em risco de desenvolver LP. Utilizaramse os testes de Kolmogorov-Smirnov, D'Agostino \& Pearson e Shapiro-Wilk para verificar a normalidade das variáveis "risco para desenvolvimento de LP", "grau de dependência para realização das AVDs" e "tempo de institucionalização"(14). Os testes mostraram tratarem-se de variáveis de distribuição não normal. Para análise inferencial, utilizou-se o 
teste não paramétrico de correlação de Spearman a fim de verificar a existência de correlação entre as seguintes variáveis: "risco para desenvolvimento de LP"; "grau de dependência para realização das AVDs"; "tempo de permanência na ILPI"; "risco para desenvolvimento de $L P$ " e "grau de dependência para realização das AVDs". Todos os testes foram realizados utilizando o software SPSS - versão 21.0. Foi adotado o nível de significância de $5 \%$ $(p<0,05)$.

O estudo obedeceu aos preceitos éticos previstos na Resolução №466/12 e foi aprovado pelo Comitê de Ética em Pesquisa (Parecer Ético no 517.957). O Termo de Consentimento Livre Esclarecido foi assinado pelo idoso ou pelo seu responsável legal. Cabe ressaltar que o responsável legal poderia ser um familiar, cuidador ou a própria instituição em que o idoso residia.

\section{RESULTADOS E DISCUSSÃO}

Dos 42 idosos em estudo, a maioria (25 $59,5 \%)$ era do sexo feminino. A média de idade desses idosos foi de 79,07 anos ( \pm 9,1 anos). A maioria (38 - 90,5\%) custeia a internação com a aposentadoria e $32(76,2 \%)$ foram internados por motivos familiares. A média do tempo de internação foi de 5,4 anos ( \pm 6,0 anos), com tempo de permanência mínimo de 06 meses e máximo de 27 anos. No que se refere à escolaridade, $17(40,5 \%)$ tinham de um a quatro anos de estudo, seguidos de 11 (26,2\%) analfabetos e quatro $(9,6 \%)$ com cinco ou mais anos de estudo. Não foi possível identificar o nível de escolaridade de 10 (23,7\%). Com relação ao número de filhos, 18 (42,9\%) não tinham filho, 20 $(47,6 \%)$ tinham um ou mais filhos e quatro $(9,5 \%)$ não informaram.

O predomínio do sexo feminino entre os idosos que residem nas ILPI estudadas reflete o panorama nacional, que aponta para o aumento da expectativa de vida dos indivíduos com preponderância do sexo feminino. Conforme o censo realizado em $2010^{(15)}$, as mulheres representam $55,5 \%$ da população idosa brasileira e $61 \%$ são idosas com mais de 80 anos de idade.

A Tabela 1 mostra o risco de os idosos participantes desenvolverem LP segundo a Escala de Braden.

Tabela 1 - Risco de os idosos em estudo desenvolverem LP segundo a Escala de Braden - Divinópolis, Minas Gerais (2014).

\begin{tabular}{lll}
\hline Nível de risco & $\mathbf{n}$ & $\%$ \\
Sem risco & 15 & 35,7 \\
Moderado & 11 & 26,2 \\
Alto & 10 & 23,8 \\
Baixo & 4 & 9,5 \\
Muito elevado & 2 & 4,8 \\
Total & 42 & 100,0 \\
\hline
\end{tabular}

Fonte: Dados da pesquisa, 2014.

Verificou-se que a prevalência de idosos em risco para desenvolver LP segundo a Escala de Braden foi de 64,3\% (IC 95\%: 48,0\% - 78,4\%). Dentre estes, $17(63,1 \%)$ apresentaram alterações significativas nos subitens "Fricção e cisalhamento" e "Atividade Física". A média da pontuação obtida pelos idosos na Escala de Braden foi de 15,7 ( $\mathrm{dp}=4,1$ pontos; IC 95\%: 14,4 17,0).

Estudo brasileiro ${ }^{(16)}$ mostrou que um dos principais motivos da institucionalização dos idosos era a dificuldade de desempenhar as AVDs. A dependência na realização das AVDs é gerada pela perda da capacidade funcional que, por sua vez, é influenciada pelo processo de envelhecimento ${ }^{(16-17)}$ Contudo, institucionalização do idoso pode potencializar a perda da capacidade funcional, uma vez que a maioria das ILPI não possuem recursos humanos e financeiros suficientes para proporcionar uma assistência voltada para a promoção do autocuidado ${ }^{(16)}$.

A Tabela 2 mostra o nível de dependência dos idosos em estudo para realização das AVDs segundo a Escala de Katz. 
Tabela 2 - Nível de dependência dos participantes para realização das AVDs segundo a Escala de Katz Divinópolis, Minas Gerais (2014).

\begin{tabular}{lll}
\hline Nível de dependência & $\mathbf{n}$ & $\%$ \\
6 & 13 & 31,0 \\
5 & 11 & 26,2 \\
4 & 5 & 11,9 \\
3 & 4 & 9,5 \\
1 & 4 & 9,5 \\
2 & 3 & 7,1 \\
0 & 2 & 4,8 \\
Total & 42 & 100,0 \\
\hline
\end{tabular}

Fonte: Dados da pesquisa, 2014.

Nota-se que a maioria (24 - 57,1\%) (IC 95\%: 41,0\% - 72,3\%) apresentou dependência para realizar cinco ou mais AVDs. Ainda, conforme a escala de Katz, 100,0\% dos idosos apresentaram dependência para tomar banho, vestir-se, usar o vaso sanitário, transferir-se de um local para o outro e na capacidade de micção e evacuação inteiramente autocontroladas, e a maioria (22 - 52,4\%) era dependente para alimentar-se. A média da pontuação obtida pelos idosos na Escala de Katz foi de 4,2 (dp = 1,9 pontos; IC 95\%: 3,6 - 4,8).

Entre os idosos avaliados no estudo, apenas $4,8 \%$ foram considerados independentes para realizar todas as AVDs. Estudo apontou que, quanto maior a dependência para realização das AVDs, maior o risco de institucionalização do idoso. Idosos com dependências leves para realização das AVDs apresentaram risco de institucionalização 1,7 vezes maior do que aqueles independentes, e os com dependência severa apresentaram risco 2,1 vezes maior, quando comparados àqueles independentes ${ }^{(18)}$. Assim, estimular a independência do idoso é importante para reduzir o risco de institucionalização. $O$ resultado de um ensaio clínico internacional aponta a implementação de intervenções em idosos que são dependentes para realizarem uma ou mais AVDs como meio eficaz de promover a independência destes ${ }^{(19)}$.

O teste de correlação de Spearman mostrou que há uma correlação negativa entre o grau de dependência para realização das AVDs e o risco de desenvolver $\operatorname{LP}\left(r_{s}=-0,74 ; p<0,05\right)$, ou seja, quanto maior a independência para desenvolvimento de AVD, menor o risco de desenvolver LP.

O aumento da longevidade e da invalidez aumenta a prevalência de LP e as complicações e gravidades decorrentes desta ${ }^{(9)}$. Neste estudo, quanto maior o grau de dependência para realizar AVD, maior o risco do idoso desenvolver $\operatorname{LP}\left(r_{s}=-\right.$
$0,74 ; p<0,05)$. Resultado semelhante foi encontrado em outros estudos ${ }^{(20-21)}$. Em estudo de coorte prospectivo com 183 idosos, 97,3\% dos idosos com LP apresentaram comprometimento para realizar as AVDs $(p<0,0001)^{(20)}$. Encontrou-se correlação positiva entre o grau de dependência para realizar as AVDs e o risco para desenvolver LP $(p<0,0001)^{(21) .}$ Assim, reforça-se a necessidade de implementar intervenções com apoio de uma equipe multiprofissional que promovam maior autonomia ao idoso institucionalizado no seu autocuidado $^{(19)}$. Para tanto, é imprescindível a presença do enfermeiro nas ILPI, uma vez que o estímulo ao autocuidado também é uma intervenção de enfermagem e é essencial para a segurança do idoso institucionalizado, pois contribui, não somente para a prevenção de lesões de pele, como também para melhoria da sua autoestima ${ }^{(3,19)}$. Para reduzir o risco de desenvolver LP, o enfermeiro deve descrever o grau de dependência individual para realizar as AVDs e iniciar cuidados de enfermagem preventivos.

Neste estudo, $47,2 \%$ dos idosos não possuíam filhos e 76,2\% foram internados por motivos familiares. Este achado pode estar relacionado com o surgimento de novos arranjos familiares. Estudo aponta que a inserção da mulher (principal cuidadora) no mercado de trabalho e dificuldades financeiras apresentadas pelas famílias são fatores que contribuem para a institucionalização do idoso. Além disso, a institucionalização ocorre no momento em que os idosos se tornam dependentes de cuidados numa proporção maior que a capacidade de os familiares cuidarem ${ }^{(16-17)}$.

Não houve correlação entre as variáveis "Tempo de institucionalização" e "Risco de desenvolver $\mathrm{LP}^{\prime}\left(r_{s}=0,12 ; \mathrm{p}=0,43\right)$ e "Nível de dependência nas AVDs $\left(r_{s}=0,02 ; p=0,85\right)$, o que permite inferir que 0 tempo de institucionalização não aumentou o risco de 
desenvolver LP e o grau de dependência para realização das AVDs.

A prevalência de risco em idosos para desenvolver LP foi de $64,3 \%$. Em outros estudos, esta prevalência foi de $49,0 \%{ }^{(17)}$ e de $38,8 \%$ de idosos em alto risco para desenvolver LP $^{(21)}$. Essa elevada prevalência pode estar relacionada ao uso de fraldas, posicionamento e deslocamento inadequado no leito, que levam o idoso à exposição dos fatores de risco na população analisada.

Percebe-se a importância de se implementarem medidas preventivas como elevar a cabeceira da cama no máximo 30 으, uso de camas e colchões especiais para redistribuir pressão, utilizar forro móvel para mover ou transferir pacientes acamados, mudança de decúbito, realização de fisioterapia motora e troca regular de fraldas ${ }^{(7)}$. Essas medidas são intervenções de responsabilidade da equipe de enfermagem e configuram-se como essenciais para garantir a segurança do paciente na prevenção de LP.

Além disso, a diminuição da mobilidade dos idosos participantes da pesquisa implicou no aumento do uso de fraldas, mesmo daqueles com controles da miç̧ão e da evacuação, o que aumenta o risco de LP devido à predisposição a um processo de maceração da pele que, se não controlado, leva a uma redução na força tensiva, tornando a pele susceptível à compressão, fricção e cisalhamento ${ }^{(1,7)}$. Este achado reforça a necessidade de conscientização da equipe assistencial quanto à importância do estímulo ao autocuidado do idoso, bem como das complicações decorrentes da exposição da pele à umidade por um maior período de tempo.

Diante do exposto, nota-se a necessidade de implantação de políticas públicas que visem a atenção à população idosa, bem como ações direcionadas ao cuidado dos familiares de modo a incentivar a permanência do idoso junto à família. Sugere-se que a assistência do cuidador seja iniciada pelas equipes de atendimento domiciliar, por meio da criação de grupos de discussão entre familiares e equipe de saúde, além da promoção de atividades físicas e culturais voltadas aos idosos com alteração da capacidade funcional, de forma a estimular o autocuidado, respeitando as limitações de cada indivíduo. Dessa forma, acredita-se alcançar a melhoraria da relação de cuidado entre a família e o idoso e diminuir as taxas de internação em ILPI.
Cabe ressaltar que a prevenção de LP é um dos seis eixos adotados pelo Programa Nacional de Segurança do Paciente, que envolve boas práticas, redução de erros e ausência de danos passíveis de prevenção a um paciente durante o processo de cuidados de saúde. Nesse contexto, é preciso que os cuidados de saúde ao idoso sejam prioridade nas ILPI, de forma a propiciar maior de segurança ao aprimorar os cuidados de enfermagem e minimizar os possíveis danos à saúde advindos da prática assistencial. Essa prática pode ser compreendida como um conjunto de valores, competências, atitudes e padrões de comportamentos individuais e coletivos que se refletem numa organização saudável e segura ${ }^{(22)}$.

A LP é um evento adverso evitável. O tratamento destas lesões é usualmente de alto custo, de longo prazo e gerador de danos aos pacientes, aos profissionais da saúde e aos hospitais. Assim, muitas ações devem ser implantadas com foco para a segurança, sobretudo, dos acamados, para prevenir a LP. A avaliação de riscos e a formação dos enfermeiros para os cuidados preventivos devem compor um bom plano de prevenção das LP. Ademais, é importante investir na formação do cuidador, seja ele formal, informal ou familiar, acerca dos cuidados relacionados à prevenção das LP $^{(23)}$. Outras ações como mudança de decúbito, manejo da dor, utilização de coxins de espuma, proteção de proeminências ósseas e manejo dos fatores extrínsecos e intrínsecos são atividades importantes para prevenção de $\mathrm{LP}^{(7,24)}$. Essas são intervenções de responsabilidade da equipe de enfermagem, e contribuem para a manutenção e recuperação do estado de saúde dos indivíduos, de modo a garantir uma assistência segura e livre de danos.

A utilização de escalas validadas direcionam o julgamento clínico do enfermeiro e facilita a tomada de decisão e a construção de planos de cuidados voltados à prevenção de LP baseados em evidência científica ${ }^{(9)}$. Escalas que estratifiquem o risco de desenvolvimento de LP e avaliem o grau de dependência para realizar as AVDs são importantes instrumentos de gerenciamento do cuidado de enfermagem e permitem a elaboração de planos de cuidados individualizados ${ }^{(18,20)}$. Neste estudo, as escalas de Katz e Braden mostraram alta consistência interna (Alpha de Cronbach respectivamente 0,81 e 0,82 ), o que nos permite recomendar a adoção 
das mesmas na prática assistencial de enfermeiros que atuam em ILPI.

Assim, ressalta-se a importância da aplicação de escalas preditivas de risco de desenvolvimento de LP nas ILPI pelo enfermeiro, de modo a propiciar um cuidado com base científica que direcione as intervenções de enfermagem para os problemas potenciais mais recorrentes que podem desencadear a formação de LP. A utilização da escala de Braden permite ao enfermeiro vislumbrar problemas relacionados ao estado nutricional, mobilidade, percepção sensorial, fricção e cisalhamento, umidade e grau de atividade física do idoso institucionalizado.

Dessa forma, o uso desse instrumento permite uma avaliação ampla e o planejamento do cuidado com vistas a garantir a segurança do paciente institucionalizado para prevenção de LP. Entretanto, estudo mostrou que o uso de escalas é importante, mas, por si só, não garante a implementação de intervenções voltadas à prevenção de $L P^{(25)}$, sendo necessário conciliar os resultados obtidos com a aplicação das escalas e o efetivo planejamento e implementação do cuidado.

Sugere-se que enfermeiros que atuam em ILPI guiem sua prática por meio de protocolos assistenciais voltados ao enfrentamento dos principais problemas que acometem os idosos, sobretudo aqueles que tratem da prevenção e tratamento de LP, bem como do estímulo ao autocuidado.

\section{CONCLUSÃO}

Os idosos institucionalizados apresentaram prevalência alta para o risco de desenvolver LP e dependência para desenvolver cinco ou mais AVDs. Evidenciou-se que, quanto maior o grau de dependência para realizar AVD, maior o risco de o idoso desenvolver LP. Não houve correlação entre o tempo de institucionalização, o risco do desenvolvimento de LP e grau de dependência nas AVDs. As escalas utilizadas apresentaram alta consistência interna na amostra em estudo.

Uma limitação foi o reduzido número de participantes. Assim, recomenda-se que o mesmo seja replicado em outras instituições de longa permanência de modo a ratificar estes resultados.

Conclui-se que idosos institucionalizados apresentam alta prevalência para o risco de desenvolver LP, o que reforça a necessidade da presença do enfermeiro nessas instituições, uma vez que este profissional desempenha importante função de avaliar o risco que esses apresentam para desenvolver LP, tanto na admissão quanto durante a permanência dos mesmos na instituição. Assim, sugere-se a realização de ensaios clínicos que busquem propor e testar intervenções para reduzir o risco de desenvolvimento de LP em idosos institucionalizados, bem como testar intervenções de enfermagem que estimulem o aumento da capacidade funcional, o que fortalecerá a prática baseada em evidência nesse campo e a construção de protocolos assistenciais voltados ao estímulo do autocuidado dessa clientela.

\section{REFERÊNCIAS}

1. National Pressure Ulcer Advisory Panel. Pressure Ulcer Stages Revised. [Internet]. Washington; 2016 [citado em 2017 jan 10]. Disponível em: http://www.npuap.org/about-us/ 2. Ganz DA, Huang C, Saliba D. Preventing falls in hospitals: toolkit for improving quality of care. [Internet]. Rockville, MD: Agency for Healthcare Research and Quality; 2013 [citado em 2017 set 20]. Disponível em: http://www.ahrq.gov/professionals/systems/hos pital/fallpxtoolkit/index.html

3. Brasil. Ministério da Saúde. Protocolo para prevenção de úlcera por pressão: protocolos para segurança do paciente. [Internet]. Brasília, DF; 2013 [citado em 2017 set 20]. Disponível em: http://www.anvisa.gov.br/hotsite/segurancadopa ciente/publicacoes

4. Paiva MCMS, Palhares VC, Avila MAG, Bocchi SCM, Fusco SFB, Lima $\mathrm{N}$, et al. Prevenção e acompanhamento de úlceras por pressão em idosos institucionalizados: relato de experiência. Anais do 8 을 Congresso de extensão universitária da Unesp; 2015 set 2; São Paulo, SP. [Internet]. São Paulo: Unesp; 2015. p. 1-5. [citado em 2017 set 20]. Disponível em: http://hdl.handle.net/11449/142553

5. Jaul E. Who determines the treatment for pressure ulcers in the elderly? Isr Med Assoc J [Internet]. 2013 [citado em 2017 out 20];15(9):512-5. Disponível em: http://www.ncbi.nlm.nih.gov/pubmed/24340845 6. Reis LA, Marinho MS, Lima PV. Comprometimento da capacidade funcional: significados para o idoso e sua família. Rev InterScientia [Internet]. jan/abr 2014 [citado em 2017 out 20];2(1):108-21. Disponível em: https://periodicos.unipe.br/index.php/interscient ia/article/view/62 
7. Mallah Z, Nassar N, Badr LK. The effectiveness of a pressure ulcer intervention program on the prevalence of hospital acquired pressure ulcers: controlled before and after study. Appl Nurs Res. 2014;(14):112-8.

http://dx.doi.org/10.1016/j.apnr.2014.07.001

8. Cavalcante MLSN, Borges CL, Moura AMFTM, Carvalho REFL. Indicators of health and safety among institutionalized older adults. Rev Esc Enferm USP. 2016;50(4):600-6. http://dx.doi.org/10.1590/S0080-

\section{9}

9. National Institute for Health and Care Excellence. Using a National Guideline to prevent and manage pressure ulcers. Nurs Manage [Internet]. 2014 [citado em 2017 out 20];21(2):18$21 . \quad$ Disponível em: https://www.ncbi.nlm.nih.gov/pubmed/24779761 10. Herdman $\mathrm{TH}$, editors. NANDA international nursing diagnoses: definitions and classification, 2015-2017. Porto Alegre: Artmed; 2015.

11. Lino VTS, Pereira SRM, Camacho LAB, Ribeiro Filho ST, Buksman S. Adaptação transcultural da escala de independência em atividades da vida diária [Escala de Katz]. Cad Saúde Pública [Internet]. 2008 [citado em 2017 out 20];24(1):103-2. Disponível em: http://www.scielo.br/scielo.php?pid=S0102-

\section{X2008000100010\&script=sci arttext}

12. Paranhos WY, Santos VLCG. Avaliação de risco para úlceras de pressão por meio da Escala de Braden, na língua portuguesa. Rev Esc Enferm USP [Internet]. 1999 [citado em 2017 out 20];33(1):191-206. Disponível em: http://www.ee.usp.br/reeusp/upload/pdf/799.pdf 13. Landis JR, Koch GG. The measurement of observer agreement for categorical data. Biometrics [Internet]. 1977 [citado em 2017 out 20];33(1):159-74. Disponível em: http://www.ncbi.nlm.nih.gov/pubmed/843571

14. Razali, NM, Yap BW. Power comparisons of shapiro-wilk, kolmogorov-smirnov, lilliefors and anderson-darling tests. J Stat Model Analytics [Internet]. 2011 [citado em 2017 out 20];2(1):2133. Disponível em: http://www.de.ufpb.br/ ulisses/disciplinas/norm ality tests comparison.pdf

15. Instituto Brasileiro de Geografia e Estatística (IBGE). Sinopse do censo demográfico de 2010 [Internet]. Rio de Janeiro; 2011 [citado em 2017 set 25]. Disponível em: https://ww2.ibge.gov.br/home/estatistica/popula cao/censo2010/default sinopse.shtm
16. Lisboa CR, Chianca TCM. Perfil epidemiológico, clínico e de independência funcional de uma população idosa institucionalizada. Rev Bras Enferm [Internet]. 2012 [citado em 2017 out 20];65(3):482-7. Disponível

em: http://www.scielo.br/pdf/reben/v65n3/v65n3a1 3.pdf

17. Aguiar ESS, Soares MJGO, Caliri MHL, Costa $M M L$, Oliveira SHS. Assessment of functional capacity of the elderly associated with the risk for pressure ulcer. Acta Paul Enferm [Internet]. 2012 [citado em 2017 out 20];25(1):94-100. Disponível em:

http://www.scielo.br/pdf/ape/v25nspe1/pt 15.pdf 18. Fong JH, Mitchell OS, Koh BS. Disaggregating activities of daily living limitations for predicting nursing home admission. BMC Health Serv Res [Internet]. 2015 [citado em 2017 out 20];50(2):560-78. Disponível em: http://www.ncbi.nlm.nih.gov/pubmed/25256014 19. Eklund K, Wilhelmson K, Gustafsson H, Landahl S, Dahlin-Ivanoff S. One-year outcome of frailty indicators and activities of daily living following the randomised controlled trial: continuum of care for frail older people. BMC Geriatr [Internet]. 2013 [citado em 2017 out 20];13:76. Disponível em: http://www.biomedcentral.com/14712318/13/76

20. Freitas JPC, Alberti LR. Application of the Braden Scale in the home setting: incidence and factors associated with pressure ulcers. Acta Paul Enferm. 2013;26(6):515-21. http://dx.doi.org/10.1590/S010321002013000600002

21. Aydin G, Mucuk S. The evaluation of daily living activities, pressure sores and risk factors. Rehabil Nurs [Internet]. 2015 [citado em 2017 out 20];40(2):84-91. Disponível em: http://www.ncbi.nlm.nih.gov/pubmed/24633702 22. Reis CT, Martins M, Laguardia J. A segurança do paciente como dimensão da qualidade do cuidado de saúde: um olhar sobre a literatura. Ciênc Saúde Colet [Internet]. 2013 [citado em 2017 out 20];18(7):2029-36. Disponível em: http://www.scielo.br/scielo.php?pid=S1413$81232013000700018 \&$ script=sci arttext 23. Silva AJ, Pereira SM, Rodrigues A, Rocha AP, Varela J, Gomes LM, et al. Custo econômico do tratamento das úlceras por pressão: uma abordagem teórica. Rev Esc Enferm USP [Internet]. 2013 [citado em 2017 out 20];47(4):971-6. Disponível em: 
http://www.scielo.br/pdf/reeusp/v47n4/0080-

6234-reeusp-47-4-0971.pdf

24. Moore Z, Stephen HJ, Callaghan R. Prevention and management of pressure ulcers: support surfaces. $\mathrm{Br} \mathrm{J}$ Nurs [Internet]. 2014 [citado em 2017 out 20];23(6):38-43. Disponível em: http://www.ncbi.nlm.nih.gov/pubmed/24690750 25. Johansen E, Moore Z, Van Etten M, Strapp H. Pressure ulcer risk assessment and prevention: what difference does a risk scale make?: a comparison between Norway and Ireland. J Wound care [Internet]. 2014 [citado em 2017 out 20];23(7):369-70. Disponível em: http://www.ncbi.nlm.nih.gov/pubmed/25041313

Nota: Este estudo faz parte de uma Monografia de Final de Curso e não contou com contribuição de agência de fomento.

Recebido em: 01/11/2017

Aprovado em: 07/03/2018

Endereço de correspondência:

Mariana Ferreira Vaz Gontijo Bernardes

UFMG - Escola de Enfermagem

Av. Prof. Alfredo Balena, $n^{\circ} 190$ - Bairro: Santa Efigênia

CEP: 30130-100 - Belo Horizonte/MG - Brasil

E- mail: marianagontijoufsj@gmail.com 